\title{
Un savoir occulté ou pourquoi le magnétisme animal ne fut-il pas pensé "comme une branche très curieuse de psychologie et d'histoire naturelle»?
}

A darkened knowledge; or why was the animal magnetism not thought as "one very peculiar part of psychology and natural history"?

\section{Nicole Edelman}

\section{(Q) OpenEdition}

Journals

Édition électronique

URL : http://journals.openedition.org/rh19/3877

DOI : $10.4000 /$ rh 19.3877

ISSN : $1777-5329$

Éditeur

La Société de 1848

Édition imprimée

Date de publication : 1 juillet 2009

Pagination : 115-132

ISSN : 1265-1354

Référence électronique

Nicole Edelman, « Un savoir occulté ou pourquoi le magnétisme animal ne fut-il pas pensé «comme une branche très curieuse de psychologie et d'histoire naturelle»? », Revue d'histoire du XIXe siècle [En ligne], 38 | 2009, mis en ligne le 05 juillet 2009, consulté le 01 mai 2019. URL : http:// journals.openedition.org/rh19/3877 ; DOI : 10.4000/rh19.3877 


\section{NICOLE EDELMAN}

\section{Un savoir occulté \\ ou pourquoi le magnétisme animal ne fut-il pas pensé "comme une branche très curieuse de psychologie et d'histoire naturelle»?}

Lorsque le magnétisme animal est théorisé et mis en pratique au début des années 1770 par le médecin autrichien Franz Anton Mesmer (17341815), les conceptions du monde et de l'être humain entrent dans une ère de forts bouleversements. En cette fin du XVIII ${ }^{e}$ siècle, le christianisme est encore certes une croyance dominante en Europe; l'existence de l'âme et la croyance en son immortalité demeurent une certitude pour beaucoup. Les hommes sont donc capables de relier sans problème conceptuel majeur la réalité tangible du monde terrestre à celle supposée des extra-mondes, palliant l'invisibilité de ces derniers par des représentations imaginaires. Philosophes et public lettré commencent cependant à chercher à expliquer la mécanique de l'esprit comme celle de la nature sans recourir à l'intervention divine; la réflexion sur les liens entre corps et esprit, sur l'unité et l'identité du moi s'amplifie ${ }^{1}$. La mise au jour du magnétisme animal s'inscrit dans cet espace conceptuel, tout comme celle du somnambulisme magnétique par le marquis de Puységur en 1784. Cette découverte (renommée hypnose en 1843) contribue, c'est du moins mon hypothèse, à ajouter de nouvelles questions sur l'articulation entre physique et moral, entre visible et invisible. Ce sommeil lucide, comme il est aussi nommé, semble en effet capable de révéler un état d'où la conscience est absente et peut-être même de dissocier l'unité du moi. Il paraitt aussi rendre poreuses les frontières de la matière et du corps. Les somnambules disent ainsi non seulement voir l'intérieur de leur corps (et se soignent) mais ils voyagent aussi dans un invisible, celui d'un extra-monde, d'un surnaturel, souvent habité d'esprits immatériels, de fantômes et deviennent des voyants ou des visionnaires que des chrétiens mettent au service de questions théologiques. Ces phénomènes en rappel-

1. On commence à pouvoir distinguer entre un niveau psychologique et un niveau métaphysique ou philosophique avec le philosophe allemand Christian Wolff (1679-1754) tandis que se développe une philosophie empiriste avec les réflexions de Thomas Hobbes, John Locke, David Hartley et David Hume. Voir Jacqueline Carroy, Annick Ohayon, Régine Plas, Histoire de la psychologie en France, XIX'eXX'siècles, Paris, La Découverte, 2006. 
lent bien d'autres mais sont en même temps différents. S'ils ressemblent par certains traits aux états de possession, ils s'en éloignent par leur calme et leur sérénité. Ils se rapprochent également des extases que connurent certain(e)s mystiques, mais le sommeil lucide touche toutes sortes d'individus quelles que soient leurs croyances. Ils se répandent enfin très largement, avant et après la Révolution française, tant en France que dans toute l'Europe. Il ne s'agit donc pas de quelques cas marginaux, même si l'ampleur de ce déploiement a été largement refoulée par les détenteurs du pouvoir spécifiquement en France - et donc longtemps ignorée des historiens. Je m'attacherai ici à comprendre les raisons de l'occultation de ce savoir magnétique, particulièrement celui du somnambulisme magnétique en évoquant d'abord l'émergence de ces découvertes puis leurs déploiements et leurs condamnations en France par les instances scientifiques, respectivement en 1784 et 1837, en distinguant nettement ces deux temps que séparent à la fois la Révolution française et la première Restauration. Je chercherai alors à analyser leurs modes de réception pour décrypter les motifs de leur mise à l'écart. Je proposerai enfin des hypothèses pour éclairer ces occultations.

\section{DE LA NAISSANCE DU MAgNÉTISME ANIMAL À SA CONDAMNATION}

Dans sa thèse de médecine, Dissertatio physico-medica de planetarum influxu (Dissertation physico-médicale sur l'influence des planètes), soutenue en 1766 à Vienne, Franz Anton Mesmer avait élaboré une théorie globale de la santé et de la maladie fondée sur la certitude d'une influence mutuelle entre les corps célestes, la terre et les corps vivants, influence rendue possible par l'existence d'un fluide subtil et universel dans lequel baignerait l'univers tout entier et qui pénétrerait tous les corps vivants à l'intérieur desquels il circulerait. Considérant que le corps humain présentait des propriétés magnétiques analogues à celles que produit l'aimant, Mesmer avait nommé «magnétisme animal » à la fois la faculté que posséderaient les êtres vivants d'être parcourus par l'omniprésent fluide et leur capacité, pour les mieux dotés, de le redistribuer. L'ensemble de cette théorie et sa mise en pratique furent nommés «magnétisme animal» ou «mesmérisme». Sachant que la mauvaise circulation du fluide était, pour Mesmer, à l'origine des maladies, le rôle du médecin (qui doit, lui-même, posséder ce fluide à suffisance), était de supprimer ces obstacles en magnétisant le corps. Le thérapeute s'approchait du patient le long duquel il passait ses mains, les arrêtant sur certains "pôles» du corps, lieux jugés névralgiques, activant ainsi la circulation du fluide, prolongeant parfois son geste d'un léger coup d'une baguette magnétisée. Au final, l'harmonieuse distribution du fluide devait se trouver rétablie et la guérison obtenue. Mesmer mit d'abord en pratique sa théorie à Vienne puis, lorsqu'il en fut chassé, la perfectionna à Paris où il arriva en 1778. Son succès y prit une 
telle ampleur qu'il inventa un baquet pour traiter plusieurs malades en même temps. Ce baquet, composé de limaille de fer et d'eau, était censé accumuler le fluide, redistribué par des tiges en fer qui en sortaient et que les malades empoignaient. Installés tout autour, dans une pénombre apaisante, au son d'une musique douce (souvent créée par Mozart au temps viennois), reliés par une corde, les riches malades - car la cure coûtait cher - formaient ainsi une chaîne humaine dans laquelle le fluide circulait tandis que Mesmer touchait les corps ici et là. La curation se terminait bien souvent par une crise convulsive, sans qu'aucune parole ne soit prononcée, une salle capitonnée étant prévue pour y déposer le patient trop agité.

En cette fin du XVIII e siècle, l'idée de fluide n'a rien d'extraordinaire à la fois parce que la notion "d'éther", remplissant l'univers, est largement admise, que Newton a montré l'existence d'une attraction universelle, que les premières montgolfières s'envolent et qu'enfin les expériences sur l'électricité débutent. Dans le monde savant et pour un public cultivé, le mesmérisme est donc parfaitement intelligible et crédible dans un cadre cependant plus proche de la physique et de l'astronomie que de la médecine. La médecine esquisse à peine sa révolution clinique et reste encore, et pour bien longtemps dans certains cas, profondément liée aux visions des siècles antérieurs : néohippocratisme, galénisme, théorie des humeurs, vitalisme se mêlent au pragmatisme de médecins et de chirurgiens qui mettent au jour une anatomie de plus en plus précise en ouvrant les corps vivants ou morts. Il faut attendre le début du XIX ${ }^{e}$ siècle pour que naissent, autour de Xavier Bichat et de François Broussais, l'anatomo-pathologie et la physiologie. Le magnétisme animal se distingue pourtant de ces théories médicales non seulement par une interprétation extrêmement simple de la santé et la maladie - à savoir la bonne ou la mauvaise circulation des fluides dans le corps - mais il permet aussi de maintenir l'inscription du corps dans un réseau d'influences cosmiques. Enfin, il s'appuie sur des liens relationnels entre les êtres humains, nécessaires à l'efficacité des soins, ces «attractions» mutuelles pouvant donner lieu à des interprétations sociales et idéologiques.

Pourtant, en 1784, la découverte du somnambulisme magnétique par Puységur ${ }^{2}$ oblige à questionner les fondements théoriques et pratiques du mesmérisme et à poser de nouvelles questions sur les rapports entre magnétiseur et magnétisé comme sur les frontières entre corps et esprit. Or c'est à cette date même que des commissions académiques formées sur ordre de Louis XVI condamnent le magnétisme animal. Le somnambulisme magnétique n'est donc alors pas en cause. 


\section{LES CONDAMNATIONS DE I784 ET LEURS MOTIFS}

Depuis l'arrivée de Mesmer à Paris en 1778, des médecins se disaient inquiets des pratiques incongrues pratiquées par ce Viennois en son hôtel parisien. Louis XVI, alerté par ces plaintes, fit convoquer deux commissions, l'une mixte formée par la Faculté de médecine et l'Académie royale des sciences et l'autre par la Société royale de médecine, afin d'enquêter précisément sur l'existence du fluide magnétique. En 1784, Mesmer avait quitté la France et ce fut l'un de ses anciens disciples, pourtant désavoué, Charles Deslon (1750-1786), docteur-régent à la Faculté de médecine de Paris, membre de la Société royale de médecine et premier médecin du comte d'Artois, qui présenta le magnétisme animal à ces savants observateurs. La première commission observa, expérimenta et finit par conclure à l'inexistence du fluide en expliquant que les effets produits, à savoir convulsions et crises, étaient dus à l'imitation, à l'imagination ou à l'attouchement. Laurent de Jussieu se dissocia de ses collègues et publia son propre rapport reconnaissant dans les effets du magnétisme, la possible action d'une "chaleur animale». À ces conclusions enfin, un rapport secret de la première commission fut envoyé au roi dénonçant le danger moral de la mixité et de la promiscuité liée aux pratiques magnétiques, en particulier pour les femmes ${ }^{3}$.

Le magnétisme était donc dénoncé à la fois comme théorie physique et comme porteur d'immoralité mais ses effets thérapeutiques, et en particulier ceux du somnambulisme, mis au jour l'année même de la réunion des commissions, en 1784, n’avaient pas été médicalement observés. Si le fluide n'existait pas, les commissions soulignaient cependant l'importance de l'imagination à laquelle les médecins d'alors donnaient une place très forte. Le médecin Virey, rédigeant en 1818 l'article consacré à ce mot dans le Dictionnaire des sciences médicales, la présente comme responsable du génie comme de fureurs, un "gouvernail suprême de la machine humaine en maladie encore plus qu'en santé» ${ }^{4}$, capable d'opérer de vrais miracles mais aussi de graves maux. Il rappelle les effets de l'exorcisme, le pouvoir thaumaturge de rois ou de saints et... «les pilules de mie de pain» qu'on n'appelle pas encore placebo $^{5}$. Il distingue cependant deux types d'imagination : celle, active, des poètes, créateurs, artistes et celle, passive, des femmes, des enfants, des vieillards qui parfois exalte et fait les martyrs. Constatant enfin qu' «aucun

3. Voir Robert Darnton, Mesmerism and the End of the Enlightement in France, Cambridge (Mass.), Harvard University Press, 1968, traduction française La Fin des Lumières : le mesmérisme et la Révolution, Paris, Perrin, 1984, p. 63-69. Franklin Rausky, Mesmer ou la révolution thérapeutique, Paris, Payot, 1977, chapitre 6, p. 123-149. Rapport des commissaires chargés par le roi de l'examen du magnétisme animal (rédigé par J.-S. Bailly), Paris, Moutard, 1784 et Rapport des commissaires de la Société royale de médecine, nommés par le roi pour faire l'examen du magnétisme animal, Paris, Impr. royale, 1784.

4. Julien-Joseph Virey, «Imagination», dans Dictionnaire des sciences médicales, Paris, Panckouke, 1818, volume 24, p. 17.

5. Idem, p. 51-52 
levier de l'économie n'étant plus puissant que l'imagination, il devient d'une souveraine importance d'apprendre à gouverner son activité et son énergie. ${ }^{6}$ Comment? Par l'étude, la culture des sciences et des lettres, la religion, la vie retirée, la bonté, la bonne conscience, le voyage, puisque si l'imagination est liée au système nerveux elle varie aussi en fonction des tempéraments, des climats, des saisons, des régimes et des habitudes...

Il semble donc que si le mesmérisme est mis à l'écart de la médecine en 1784 , ce n'est pas d'abord pour des raisons médicales, puisque les commissions constataient que le fluide salvateur n'existant pas, l'efficacité éventuelle du magnétisme pouvait tout de même être rapprochée des "médications en mie de pain" ou... de l'imagination. Des recherches auraient pu alors aller plus avant pour s'enquérir de ces effets et de ceux de la "chaleur animale» dont parlait Jussieu. La Révolution arrête toute recherche de ce type. En revanche, on craint le charlatanisme : les caricatures de la fin du XVIII siècle représentent volontiers les magnétiseurs, médecins ou non, avec des bonnets d'ânes ou en ânes tout entier, se gaussant du public qui gobe ces charlataneries, de surcroît fort coûteuses. Ces plaisanteries visent bien sûr à ridiculiser cette nouvelle pratique et sans doute plus encore à disqualifier le magnétisme. Celui-ci proposait en effet une compréhension et un traitement des maladies si simple et si facile à saisir qu'il pouvait rendre inutile le savoir des médecins. Pourtant tous les médecins praticiens du magnétisme défendaient au contraire la nécessité d'être docteur en médecine pour comprendre les maux et trouver les points sensibles à traiter. Ils plaidaient donc pour l'inclusion de ces connaissances et de ces traitements dans la science médicale afin d'en écarter les charlatans. À lire les comptes rendus des commissions et les réactions qu'elles suscitent, d'autres raisons à ce rejet du magnétisme en 1784 peuvent donc apparaître. Je proposerai quelques hypothèses.

Le magnétisme animal est tout d'abord profondément marqué par l'ambiguïté de son créateur. Franz Anton Mesmer qui fut représenté, dès son vivant, à la fois comme un médecin mais aussi comme un séducteur avide d'argent. À Vienne, il avait un très bel hôtel dans les dépendances duquel il pouvait recevoir ses riches patients et patientes. Ami de Mozart (et francmaçon comme lui), il donnait concerts et bals et menait une brillante vie mondaine grâce à la fortune de son épouse. Et s'il avait quitté la capitale impériale, c'est en raison du scandale causé par l'étrange cure qu'il avait proposé à une jeune aristocrate, pianiste et aveugle, fille d'un conseiller impérial, Maria-Theresa von Paradis. Or, les soins qu'il distribue à Paris coûtent toujours très chers et sont donc perçus comme un luxe réservé à une clientèle riche et oisive, au moment même où d'autres savants adoptent et diffusent les Lumières de la raison et celles de la révolution scientifique. Beaucoup stigmatisent ainsi à la fois le caractère aristocratique des cures magnétiques 
et leur mixité, en particulier autour du baquet, que des caricatures montrent comme des séances fort libertines. Les crises convulsives nécessitent en effet bien souvent de dégrafer les corsets des femmes tombées en pâmoison avant de les porter dans la chambre capitonnée destinée à cet effet. Enfin, et cela me paraît important, Mesmer est perçu comme autrichien et la haine que suscitait la reine Marie-Antoinette, l'Autrichienne, a pu l'englober d'autant qu'elle était, elle aussi, accusée de frasques sexuelles.

Pourtant la critique hostile au magnétisme animal se déploie plus nettement encore après la Révolution française et le premier Empire, et c'est au moment de la première Restauration que les anti-mesmériens se déchaînent, alors que le somnambulisme magnétique, qui n'avait pas été examiné par les commissions de 1784, est bien implanté dans toute l'Europe ${ }^{7}$.

\section{L'AMPLEUR DU DÉPLOIEMENT CRITIQUE ET LA RÉCEPTION DU SOMNAMBULISME MAGNÉTIQUE \\ (PREMIÈrE MOITIÉ DU XIX SIÈCLE)}

Amand Marc Jacques de Chastenet, marquis de Puységur, avait donc découvert en 1784 cet état modifié de conscience qu'il appelle somnambulisme magnétique. Ce sommeil lucide pose bien des questions au monde savant, aux physiciens, médecins et philosophes : qu'advient-il en effet à l'être humain mis dans cet état? À quoi sont dues les capacités qu'il semble alors acquérir? Est-ce ce sixième sens tant rêvé qui serait enfin mis au jour? Ce somnambulisme artificiel a en effet pour conséquence immédiate de permettre à la voyance de renaître ${ }^{8}$. Longtemps adossée à l'astrologie et aux horoscopes dont la validité scientifique avait été mise à bas par les découvertes de Copernic et de Galilée, la voyance renaissante se fonde pour presque un siècle sur le somnambulisme magnétique qui sera sa caution scientifique. Des somnambules, très majoritairement des femmes, disent avoir le somnambulisme et pouvoir ainsi voir le passé et l'avenir, tout comme l'intérieur des corps, devenant voyantes et guérisseuses mais aussi visionnaires. Brouillant les frontières entre corps et esprit, le somnambulisme peut en effet laisser croire qu'il permet de communiquer avec l'au-delà et suscite bien des croyances spiritualistes qui se déploient largement après la Révolution française et qui se ravivent dans le romantisme, tant allemand ${ }^{9}$ d'abord que français ensuite. Le somnambulisme magnétique devient à la fois un moyen de communication

7. Seule l'Angleterre reste à l'écart jusqu'aux années 1830 , sans doute parce que le magnétisme animal et surtout le somnambulisme magnétique sont perçus comme français et donc dangereux. Voir Alison Winter, Mesmerized. Powers of Mind in Victorian Britain, Chicago, The University of Chicago Press, 1998.

8. Voir Nicole Edelman, Voyantes, guérisseuses, visionnaires en France, 1785-1914, Paris, Albin Michel, 1995.

9. Voir l'article de Luis Montiel dans ce numéro. 
avec le divin pour des chrétiens en quête de voies mystiques ${ }^{10}$ et le support de nouvelles croyances pour ceux qui ne parviennent à résoudre l'aporie entre la philosophie du progrès et les échecs des espoirs révolutionnaires qu'en se tournant vers l'invention de théologies rationnelles.

En 1817, dans La Philosophie de l'esprit, troisième tome de L'Encyclopédie des sciences philosophiques ${ }^{11}$, Hegel examine l'état magnétique et les facultés somnambuliques qui en découlent. Il dépasse largement les certitudes des médecins magnétiseurs puisqu'il accepte l'hypothèse d'une capacité de clairvoyance chez certains individus malades. Il considère en effet le somnambulisme comme une maladie de l'âme qui permet d'échapper au pouvoir de la conscience. Lâme acquiert alors de facto un certain nombre de pouvoirs qui ne relèvent pourtant en aucun cas d'une activité supérieure de l'esprit, bien au contraire. Pendant le somnambulisme magnétique, «l'activité des organes dirigés vers le dehors, passe dans les organes internes [...] la conscience est rabaissée à la naturalité simple [...]. Pour cette raison, l'activité efficiente de l'âme, pendant le somnambulisme magnétique, échoit [...] au cerveau du système de reproduction, à savoir aux ganglions, ces nouds complexes des nerfs de la partie inférieure du corps $»^{12}$. On retrouve là les interprétations de la médecine romantique allemande. Cette sensibilité particulière explique que le somnambule puisse ressentir et percevoir sans la médiation du sens de la vue, parfois par l'épigastre. Des hommes, ajoute Hegel, sont aussi capables de détecter du métal, du sel ou de l'eau se trouvant sous la surface du sol. Le philosophe ajoute que cette capacité a permis de retrouver des voleurs, des meurtriers ou des cadavres. D'autres phénomènes consistent «en ce que sans le concours d'un sens spécifique quelconque [...] naissent, d'une sensation indéterminée, un pressentiment ou une vue, une vision de quelque chose qui n'est pas sensiblement proche, mais éloigné dans l'espace ou dans le temps, de quelque chose de futur ou de passé» ${ }^{13}$. Pour Hegel, en effet l'âme individuelle est la partie d'un tout universel. «L'âme est ce qui pénètre tout, qui n'existe pas simplement dans un individu particulier. $»^{14}$ Dans certaines conditions, l'âme d'un individu peut donc ne plus être liée à l'espace qui l'entoure. Ce don de seconde vue "n'a rien de rare", estime-t-il. L'âme voyante est alors même capable de "s'élever au-dessus de la condition du temps", au moins en ce qui concerne le passé. L'âme peut «à nouveau se rendre présent quelque chose que le temps écoulé a complètement fait sortir de sa conscience éveillée » ${ }^{15}$. Hegel n'exclut pas non plus la clairvoyance du futur. Il reconnaît enfin la capacité du somnambule à la fois à éprouver ce que ressent un autre

10. Voir l'article de Christine Bergé dans ce numéro.

11. Georg Wilhelm Friedrich Hegel, Encyclopédie des sciences philosophiques, tome III, Philosophie de l'esprit (1817), texte présenté, traduit et annoté par Bernard Bourgeois, Paris, Vrin, 1988.

12. Idem, p. 486.

13. Idem, p. 475.

14. Ibidem.

15. Idem, p. 480 et p. 478. 
qui lui est proche (maladie, sentiments...) et à déterminer avec justesse la nature et le cours de ses propres maux. Hegel souligne enfin l'extrême dépendance et les liens étroits du magnétiseur, un être fort, et de «la personne magnétique", un individu plus faible, donc souvent une femme. La certitude du philosophe concernant l'existence de ces phénomènes de clairvoyance est donc patente tout comme leur caractère pathologique.

Autour de la France, depuis 1815, la Russie, la Prusse et le Danemark ont ainsi admis, certes avec difficulté, la réalité de ces phénomènes et certaines chaires de médecine y sont confiées à des médecins pratiquant le magnétisme. En France, de nombreux médecins sont partie prenante de ces phénomènes; des cures par magnétisme continuent d'être pratiquées, en particulier par les médecins des eaux thermales ${ }^{16}$ et d'autres expérimentent les effets du somnambulisme artificiel dans des grands hôpitaux parisiens. Les positions sont donc loin d'être univoques et évoluent beaucoup au fil des années.

Après celles de Hegel, je rappellerai rapidement les conceptions de Puységur, découvreur du somnambulisme, qui non seulement n'est pas médecin, mais dont les conceptions médicales «neurologiques» demeurent largement celles du XVIII siècle. Le marquis de Puységur écrit ainsi : "Dès lors qu'ils [les médecins] s'accordent tous à penser que le cerveau est le réservoir commun des esprits animaux, d'où par le canal des nerfs, ils se répandent dans toutes les parties du corps, pour y entretenir le mouvement et la vie, ne s'ensuit-il pas nécessairement que parmi tous les moyens puissants dont la nature se sert pour maintenir notre existence, le plus efficace doit être celui qui, agissant immédiatement sur le cerveau, n'y reporte ou n'y entretient que la quantité d'esprit nécessaire à l'exercice de ses fonctions [...].» ${ }^{17}$ Puységur évoque encore l'existence dans l'homme d' "une essence incorporelle et intelligente, dont le sens somnambulique est l'éclatante, quoique bien faible manifestation ${ }^{18}$. Le sommeil naturel ou le sommeil somnambulique sont donc, pour lui, seuls capables de refaire circuler harmonieusement ces esprits animaux. Le sommeil magnétique « réactive les esprits animaux dans le cerveau et rend à ce dernier, dont il rétablit les ressorts, toute l'énergie nécessaire à l'exercice de ses fonctions» ${ }^{1}$. Par ailleurs, pour Puységur, la cause du magnétisme n'est pas un fluide, ni un courant particulier mais elle est dans l'homme "soumise à l'empire et à la direction de sa volonté» ${ }^{20}$. Pourtant, le marquis pense que seul la physique peut permettre de prouver l'existence de ce magnétisme dont il rappelle qu'il n'a d'efficacité que lorsqu'il est pratiqué avec compassion et concerne un malade. Celui-ci dispose alors «d'un sens intérieur qui se déve-

16. Voir les articles de Jacqueline Carroy et de Jan Goldstein.

17. Amand Marc Jacques de Puységur, Un somnambule désordonné? Journal du traitement magnétique du jeune Hébert, édition établie et présentée par Jean-Pierre Peter, Le Plessis-Robinson, Synthélabo, 1999, p. 155.

18. Idem, p. 154.

19. Idem, p. 156.

20. Idem, p. 303. 
loppe en lui et il peut connaître et juger sciemment de la nature des maladies et des moyens secondaires à employer pour la guérir» ${ }^{21}$.

Quant à l'existence ou la non-existence du fluide, si elle a été niée par les commissions de 1784, elle continue d'être discutée par les magnétiseurs, médecins ou non (qu'on divise, trop rapidement me semble-t-il, entre fluidistes et spiritualistes). Je présenterai donc la conception de deux médecins ${ }^{22}$ ayant pratiqué des soins par somnambulisme magnétique pour éclairer cette discussion.

Le premier, Étienne Georget (1795-1828), médecin hospitalier à La Salpêtrière, disciple d'Esquirol, pose la question si présente au début du XIX ${ }^{e}$ siècle des rapports du physique et du moral, du corps et de l'esprit, à propos des folles et des hystériques non aliénées qu'il soigne. Pratiquant le somnambulisme magnétique sur ces femmes, il est convaincu que ces phénomènes "prennent leur source dans la vie cérébrale» ${ }^{23}$. Ses somnambules sont, comme toutes leurs consœurs, capables de se prescrire des médicaments simples (qu'elles connaissent bien puisqu'elles sont hospitalisées ${ }^{24}$ ), d'annoncer longtemps à l'avance et avec précision la date, l'heure et la durée précise de leurs crises, ce qui est une constante des cures somnambuliques. Georget note cependant que "les cerveaux irritables et malades sont plus susceptibles de tomber en somnambulisme ${ }^{25}$ que les sains. Il privilégie la volonté puisqu'il pense qu'il est nécessaire que magnétiseur et somnambule "veuillent que le somnambulisme soit déterminé » ${ }^{26}$. Étienne Georget attribue la possibilité de l'existence du somnambulisme à un fluide, ou du moins un "agent de communication" émanant du cerveau, naissant de l'action cérébrale ${ }^{27}$. Il conçoit donc la possibilité de l'existence d'un lien entre deux cerveaux, assez matériel, semble-t-il. Ce lecteur de Charles Bonnet, cet élève de Pinel, ce proche d'Esquirol, ce disciple de Gall dont il pousse plus avant les travaux puisqu'il travaille sur l'ensemble du système nerveux (et non plus seulement sur le cerveau) et de ses fonctions, confirme dans un premier temps les conditions

21. Idem, p. 303.

22. Pour les conceptions d'Antoine Despine, voir l'article de Jacqueline Carroy dans ce numéro.

23. Étienne Georget, De la physiologie du système nerveux et spécialement du cerveau. Recherches sur les maladies nerveuses en général et en particulier sur le siège, la nature et le traitement de l'hystérie, de l'hypochondrie, de l'épilepsie et de l'asthme convulsif, Paris, Baillière, 2 volumes, 1821, tome 1, p. 277. Dechambre a ridiculisé ces expériences dans un article de septembre 1835 dans la Gazette médicale de Paris. Georget a certes été dupé par Pétronille et Manoury (dite Braguette) mais faut-il pour autant nier toutes les expériences de Georget? Je ne le crois pas. Voir aussi l'analyse de Jacqueline Carroy, Hypnose, suggestion et psychologie. L'invention de sujets, Paris, PUF, 1991.

24. Il s'agit de saignées, sangsues, bains, vésicatoires, moxas, peu de potions et de tisanes.

25. Étienne Georget, De la physiologie..., ouv. cité, tome 1, p. 286.

26. Idem, p. 290. Georget dit avoir magnétisé efficacement à distance.

27. Sa réflexion sur le fonctionnement du cerveau le conduit à contester, dans son ouvrage De la folie (Paris, Crevot, 1820), le mécanisme "sympathique" allant des passions au cerveau, mis en œuvre par Pinel et Esquirol. Il défend alors l'idée d'une nature idiopathique cérébrale de la folie. Du même coup il lui paraît absolument nécessaire de démarquer la folie des délires symptomatiques, ce que confirme la thèse d'Antoine Laurent Jéssé Bayle en 1822. Il distingue ainsi la psychiatrie et la neuro-psychiatrie, ce qui l'oblige à nuancer le mécanisme du rapport entre corps et esprit. 
matérielles de la pensée. Dans sa Physiologie du système nerveux, exprimant son opposition aux «subtilités métaphysiques qui ont si longtemps entravé la marche des sciences d'observation", il rejette avec force "ces causes occultes, ces principes substantiels, distincts de l'organisme, dont tant de physiologistes ont abusé pour expliquer les phénomènes des êtres organisés » ${ }^{28}$. Dans un deuxième temps, il paraît cependant déstabilisé par les étranges capacités de clairvoyance que lui révèle le somnambulisme magnétique. Dans son testament, écrit en 1826, deux ans avant sa mort, Étienne Georget écrit ainsi : "[À] peine avais-je mis au jour la physiologie du système nerveux que de nouvelles méditations sur un phénomène bien extraordinaire, le somnambulisme, ne me permirent plus de douter de l'existence en nous et hors de nous d'un principe intelligent, tout à fait différent des existences matérielles. Ce sera si l'on veut, l'âme et Dieu. Il y a chez moi à cet égard une conviction profonde, fondée sur des faits que je crois incontestables. ${ }^{29} \mathrm{On}$ a souvent lu ce texte comme une rétractation de Georget d'un matérialisme dont on l'avait accusé. J'y lirai aussi la possibilité de concevoir des compétences exceptionnelles de l'être humain, exprimées dans sa formule qui qualifie cet état : "Ce sera si l'on veut, l'âme et Dieu" (c'est moi qui souligne), brouillant ainsi les frontières entre matérialisme et spiritualisme, entre corps et esprit.

Je prendrai pour deuxième exemple celui du médecin Alexandre Bertrand, dont les conceptions sont subtiles et complexes. Ce médecin, reçu à l'École polytechnique en 1814, renvoyé au retour des Bourbons, proche de Pierre Leroux, journaliste au Globe, estime en effet que l'entrée en somnambulisme n'est liée ni à un fluide qui modifierait l'état pathologique éventuel du patient, ni à la volonté du magnétiseur. C'est le magnétisé lui-même, soumis à l'idée du magnétisme, qui modifie seul son état physiologique. La confiance que lui donne la rémission accordée par l'état de somnambulisme, qui suspend les douleurs, les inquiétudes ou même les angoisses, met en route espérance et imagination qui agissent à leur tour sur l'état physiologique et $a$ fortiori psychologique. Il considère en effet que l'être humain dispose d'une vie organique inconsciente mue par une force vitale qui le nourrit, le fait respirer, qu'on peut rapprocher du système nerveux ganglionnaire, qui serait en action dans l'état somnambulique. Cette force permet l'exaltation de la sensibilité qui est ainsi portée vers les organes intérieurs. Ces conceptions sont proches de celles des médecins romantiques allemands qui ne concevaient pas non plus une coupure entre corps et esprit, entre moral et physique, mais au contraire un "corps-esprit» ${ }^{30}$. Bertrand met en exergue ce qu'il appelle l'inertie morale du somnambule, à savoir la diminution de la force d'attention et de la faculté de réflexion, comparable à ce qui se passe dans le sommeil. La parole d'un interlocuteur, généralement du magnétiseur mais pas exclusive-

28. Archives générales de médecine, 1828, première série, $\mathrm{n}^{\circ} 7$, p. 322.

29. Idem, p. 155. Les italiques sont de l'auteur.

30. Voir l'article de Luis Montiel dans ce numéro. 
ment, peut alors le fixer sur un but précis, le rendant susceptible de ce que nous appellerions «suggestion». Bertrand admet la possibilité d'une communication de pensée entre magnétiseur et somnambule. Il parle alors de "sympathie». "Dans un degré élevé de somnambulisme, le cerveau se trouve dans un état d'exaltation assez considérable pour que les malades ressentent, par une espèce de sympathie, les impressions qui ont lieu dans le cerveau des personnes avec lesquelles ils sont en rapport. ${ }^{31} \mathrm{Il}$ souligne aussi l'importance de l'imagination, qu'il définit comme «la faculté de recevoir des objets absents, la même impression que s'ils étaient présents» ${ }^{32}$. Bertrand attribue donc aux somnambules une capacité particulière à influencer leur fonction organique. Il rappelle d'ailleurs l'existence de cette possibilité dans l'état ordinaire (ce que nous appelons un effet placebo). Il avance donc l'hypothèse de la possibilité d'une influence des somnambules sur leur corps et l'idée que les prédictions qu'ils font souvent sont simplement, mais réellement, la cause de l'effet produit. Ces effets sont prédéterminés, ainsi les annonces de crises, de douleurs ou au contraire de rémissions. Il explique et comprend le rapport particulier qui se noue entre magnétiseur et somnambule : si le somnambule a été magnétisé individuellement, sa capacité d'attention et de réflexion se trouve alors réduite et il n'entend alors que son magnétiseur et seulement quand il s'adresse à lui. En revanche, s'il est magnétisé dans un cercle de personnes, le somnambule percevra l'ensemble de cet entourage. Bertrand interprète la faculté qu'ont les somnambules d'obéir à la volonté de leur magnétiseur sans qu'il profere une parole comme une réflexion de la pensée du magnétiseur dans la tête du magnétisé, une communication de pensée grâce à l'extrême sensibilité du cerveau du somnambule. Il n'y a donc pas une action de la volonté du magnétiseur mais une connaissance de sa pensée par le somnambule qui sera d'autant plus accessible que le magnétiseur accompagnera sa pensée d'un geste qui accentuera encore le «mouvement des fibres cérébrales " ${ }^{33}$. Le somnambule très réceptif croit à la volonté du magnétiseur mais en réalité ne fait qu'agir lui-même sur lui-même.

Sur les bases explicatives proposées par ces médecins, le magnétisme et ses effets donnent donc lieu à des débats qui ne semblent cependant pas entrer en conflit avec le regard clinique naissant. En ce tout début du XIXe siècle, Xavier Bichat (1772-1802), par la dissection systématique de cadavres, met au jour en effet l'importance des tissus (membranes) qui forment à son avis l'espace anatomique et entrent dans la structure des organes. Il met ainsi en exergue une anatomo-pathologie qui fait de la vie un ensemble des propriétés

31. Alexandre Bertrand, Traité du somnambulisme et des différentes modifications quill présente, Paris, Dentu, 1823, p. 246.

32. Alexandre Bertrand, Du magnétisme animal en France, Paris, Baillière, 1826, p. 411. Il note ainsi : «Quant au somnambule de M. de Puységur, rien ne nous indique qu'il n’ait pas eu l'imagination montée par ce qu'il aura entendu raconter à son seigneur des effets d'un remède qui devait être bien merveilleux puisqu'il se donnait la peine de l'administrer lui-même. », p. 400.

33. Alexandre Bertrand, Traité du somnambulisme..., ouv. cité, p. 282. 
vitales qui résistent au processus de la mort, reliant dorénavant étroitement le normal et le pathologique. Puis François Broussais (1772-1838) affirme que les maladies sont des perturbations de l'organisme dont la connaissance relève de la physiologie. Quant aux discussions sur le cerveau inaugurées par Vicq d'Azyr, Cabanis, Gall et Spurzheim ou encore Flourens..., l'existence du somnambulisme ne fait qu'ajouter à leur complexité. Alexandre Bertrand propose ainsi un cours sur le magnétisme animal d'août 1819 à janvier 1820, tandis que des expériences sur ce thème se multiplient dans des hôpitaux parisiens et certaines avec succès : le magnétisme et le somnambulisme produisent en particulier des bienfaits analgésiques et anesthésiques ${ }^{34}$, la réduction de douleurs chroniques ou non, la disparition de paralysies (sans doute d'origine hystérique), de diverses maladies dites nerveuses et de bien d'autres types de mal-être qui relèveraient actuellement de traitements psychiatriques, de psychothérapie ou de psychanalyse ${ }^{35}$. Aussi, au vu de ces expériences, le Dr Pierre Foissac rédige-t-il une requête aux membres de l'Académie des sciences et de l'Académie royale de médecine pour qu'ils réexaminent le magnétisme ${ }^{36}$. Il souligne que le magnétisme se rattache tant à la médecine "qu'on n'aurait jamais dû l'en séparer, il devrait même être considéré comme une branche fort importante de la physiologie et de la thérapeutique. En effet nul individu n'est susceptible de somnambulisme, s'il n'est actuellement malade, ou d'une constitution extrêmement faible et délicate " ${ }^{37}$. Ce rapport, lu à l'Académie de médecine en octobre 1825, engage un débat pour savoir si une enquête sur ce thème doit être réouverte ou non. Or, au même moment, le 19 octobre, un arrêté du Conseil général des hospices interdit l'usage de tout remède nouveau qui n’aurait pas été approuvé par une commission nommée par ce même conseil ${ }^{38}$. Cette décision a-t-elle modifié l'attitude des académiciens? Toujours est-il qu'ils proposent en décembre un nouvel examen du magnétisme, qui constitue bien sûr un "remède nouveau ", et en février 1826, l'Académie royale de médecine nomme une commission essentiellement composée de médecins hospitaliers ${ }^{39}$ qui, en juin 1831, conclut d'une manière très nuancée et circonstanciée selon les cas examinés, mais globalement elle encourage à poursuivre ces recherches sur le magnétisme animal

34. Ainsi l'opération réalisée par Jules Cloquet le 8 avril 1829 sur une femme âgée de 64 ans d'un cancer du sein, magnétisée plusieurs fois par Chapelain avant l'opération puis pendant : la tumeur est enlevée "pendant tout ce temps la malade a continué à s'entretenir tranquillement avec l'opérateur et n'a pas donné le plus léger signe de sensibilité» dans Rapport sur les expériences magnétiques faites par la commission de l'Académie royale de médecine lu dans la séance des 21 et 28 juin 1831, p. 37. La patiente est laissée encore 48 heures en somnambulisme puis réveillée.

35. Cure d'Estelle par le Dr Antoine Despine ou de Lady Lincoln par les médecins Koreff et Wolowski.

36. Dr Pierre Foissac, Mémoire sur le magnétisme animal adressé à Messieurs les membres de l'Académie des sciences et de l'Académie royale de médecine, Paris, Didot, août 1825.

37. Idem, p. 5.

38. Ce que les historiens n'ont jusqu’à présent, à ma connaissance, pas relevé.

39. Formée de Bourdois de la Mothe, Double, Itard, Guéneau de Mussy, Guersant, Fouquier, Laennec remplacé par Husson, Leroux, Magendie, Marc et Thillaye. 
"comme une branche très curieuse de psychologie et d'histoire naturelle " ${ }^{40}$. Pourtant ces conclusions positives sont dans un premier temps mises de côté, n'étant ni approuvées par les Académies des sciences et de médecine, ni réfutées. Puis, en 1833, le médecin Frédéric Dubois (d'Amiens), radical adversaire du magnétisme, publie un ouvrage qui commente les expériences de cette commission en les caricaturant et les ridiculisant; il conclut à la duperie au mieux, au charlatanisme au pire ${ }^{41}$. Chargé en 1837 de former une autre commission devant observer ces phénomènes, il avance systématiquement les nouvelles normes de la révolution scientifique, qui exige une stricte répétitivité des expériences, ce qui s'avère impossible avec un «matériel » humain qui se fatigue, échoue et parfois simule... La conclusion est alors sans nuance : la commission nie l'existence même du somnambulisme magnétique. Enfin en octobre 1840, l'Académie royale de médecine se dit lassée de ces questions et annonce qu'elle refusera dorénavant de traiter du magnétisme animal qu'elle compare à la quadrature du cercle!

\section{LA QUESTION DU REFUS DU SOMNAMBULISME MAGNÉTIQUE : HYPOTHÈSES}

Ce refus pose problème ${ }^{42}$. Il est en effet difficile de comprendre la négation de l'état somnambulique, d'autant que la communauté médicale européenne était loin de toujours partager ce point de vue et que les interprétations proposées par certains, on vient de le lire, étaient largement recevables par le monde savant au vu des savoirs existants. Je ferai donc un certain nombre d'hypothèses pour comprendre cette prise de position académique.

Dans ces premières décennies du XIX ${ }^{\mathrm{e}}$ siècle, l'état somnambulique donne lieu à de multiples interprétations dont certaines le mettent radicalement à l'écart de la science, qu'elle soit médicale ou physicienne, même si, par ailleurs, médecins et physiciens s'y intéressent et ouvrent de réelles pistes de compréhension.

Tout d'abord, le somnambulisme magnétique permet à des formes de voyance de resurgir en Europe. La clairvoyance supposée que provoque cet état rend en effet crédible cette pratique en lui donnant une validité scientifique qu'elle avait perdue au XVII e siècle avec la disqualification de l'as-

40. Rapport sur les expériences magnétiques faites par la commission de l'Académie royale de médecine lu dans la séance des 21 et 28 juin 1831, 81 pages manuscrites.

41. Frédéric Dubois (d'Amiens), Examen historique et raisonné des expériences prétendues magnétiques faites par la commission de l'Académie royale de médecine pour servir à l'histoire de la philosophie médicale au XIX' siècle, Paris, Deville Cavelin, 1833.

42. Les hypothèses que je propose ne se situent pas dans la perspective "guerrière» développée par Bertrand Méheust dans son livre Somnambulisme et médiumnité, tome 1: Le Défi du magnétisme, "Les Empêcheurs de penser en rond", Le Plessis-Robinson, Synthélabo, 1999. Les oppositions sont, me semble-t-il, beaucoup plus nuancées; magnétisme et somnambulisme continuent d'être pratiqués par bien des médecins en France et en Europe au XIX ${ }^{\mathrm{e}}$ siècle. 
trologie ${ }^{43}$. Des voyantes, puisqu'il s'agit essentiellement de femmes ${ }^{44}$ qu'on nomme alors "somnambules", ouvrent boutique dans les villes et les bourgs dès le retour au calme politique en France et en Europe. Ces figures font florès dans les romans d'Honoré de Balzac, d'Alexandre Dumas, de Théophile Gautier, de Charles Nodier ou encore de George Sand. Dans Le Cousin Pons, la voyante Mme Fontaine ressemble à la célèbre Marie Anne Adélaïde Lenormand, dite Mlle Lenormand, plus douée même que son modèle puisqu'elle fait fortune dans Les Comédiens sans le savoir. Si leurs consœurs sont moins exceptionnelles, elles vendent au mieux leurs pseudo-compétences, accumulant des références pompeuses et absconses. Elles se proclament volontiers tout à la fois diplômées de sciences occultes et somnambules de naissance, disant qu'elles ont le somnambulisme. Enfin si la voyance est un métier «en chambre", elle se donne aussi à voir dans les réceptions des bonnes maisons et les salons à la mode invitent des magnétiseurs et leurs somnambules. Et certains de ces couples donnent de véritables spectacles de théâtre ou de rue où les effets anesthésiques du somnambulisme sont mis en scène : bras transpercés, membres brûlés. Les contractures provoquées y sont théâtralisées en montrant des postures incroyables du corps. D'autres couples se forment, plus inquiétants pour la science médicale cette fois, comme celui d'un médecin ou un officier de santé et d'une somnambule : celle-ci voit les maladies dans les corps, celui-ci prescrit, et tous deux échappent ainsi à l'exercice illégal de la médecine!

Si les somnambules se font guérisseuses, d'autres deviennent visionnaires et ces pratiques inquiètent alors les autorités catholiques. Ces femmes (la très grande majorité) affirment en effet converser avec les anges, les saints, parfois même avec la Vierge et Dieu en personne, plus souvent encore avec des disparus. De plus, certains croyants (et non des moindres) questionnent ces somnambules-visionnaires sur des problèmes théologiques, notant, diffusant et publiant leurs réponses

Cette résurgence de la voyance et cette manière de renouer avec un surnaturel rénové s'accompagnent, dans la première moitié du XIX siècle, du déploiement d'une véritable nébuleuse de croyances. Dans son livre sur le mesmérisme et la Révolution ${ }^{45}$, Robert Darnton relie ainsi étroitement la "fin des Lumières» à l'émergence des courants spiritualistes issus du mesmérisme après la Révolution. L'historien estime que le magnétisme animal et ses effets participent à la résolution de la question à laquelle sont confrontés bien des penseurs des Lumières après la Révolution et la Terreur. Prenant pour exemples le physiocrate Dupont de Nemours d'une part et Condorcet de l'autre, il oppose les deux solutions que ces hommes font alors émerger, le recours à un spiritualisme et à l'élaboration d'une théodicée naturelle pour

43. Voir Nicole Edelman, Histoire de la voyance et du paranormal, Paris, Le Seuil, 2006.

44. Voir Nicole Edelman, Voyantes..., ouv. cité.

45. Robert Darnton, La Fin des Lumières..., ouv. cité. 
le premier ${ }^{46}$ et, pour le second, une croyance dans le Progrès qui triomphera de la superstition.

La multiplication de spiritualismes éclectiques est en effet un des caractères de ces premières décennies du XIX ${ }^{\mathrm{e}}$ siècle. Ces croyances, portées et diffusées par des hommes (et quelques femmes) éduqués, affirment toutes l'existence d'un monde habité d'esprits et parcouru par des fluides invisibles porteurs de vertus thérapeutiques. Dans Paris, capitale du XIXe siècle, Walter Benjamin avait déjà relevé ce caractère et noté ces phrases de Philibert Audebrand écrite en 1861, décrivant l'époque de Saint-Simon dont ce souvenir précis avait donc été conservé jusqu’à cette date : «Indépendamment de la Nouvelle-Jérusalem, d'Emmanuel Swedenborg, professée par le baron Portal... il y avait le phalanstère de Charles Fourier; il y avait aussi la prétendue Eglise française de l'abbé Châtel, primat des Gaules; il y avait la restauration de l'ordre des Templiers, organisée par M. Fabré-Palaprat, il y avait le culte de l'Evadamisme, inventé par le Mapah. ${ }^{47}$ En effet, des francs-maçons mystiques, tels Jean-Baptiste Willermoz (1730-1824) ou Louis Claude de Saint-Martin (1743-1803) ${ }^{48}$ pensent que le magnétisme et le somnambulisme magnétique sont une voie d'accès privilégiée aux mondes spirituels. "Au cours des années 1780, Saint Martin se passionne pour les états somnambuliques dans lesquels il croit voir un accès à la condition de l'Adam primordial», explique Christine Bergé ${ }^{49}$. S'il abandonne ensuite ce moyen, Willermoz en revanche use largement de somnambules pour trouver des réponses sur ces au-delà et leurs habitants. Les années 1830 sont celles d'une véritable ébullition intellectuelle et religieuse : les écrits de Swedenborg sont remis en lumière et commencent à être traduits en français. Jacques François Etienne Le Boys des Guays (1794-1864) ouvre en 1835 un culte public swedenborgien; de 1838 à 1848 , il publie La nouvelle Jérusalem, revue religieuse et scientifique et traduit régulièrement les œuvres de Swedenborg. Le magnétiseur Alphonse Cahagnet se veut «spiritualiste libre de l'école de Swedenborg et de Saint Martin " ${ }^{50}$ et publie avec sa somnambule Adèle Maginot Les Arcanes de la vie future dévoilés ${ }^{51}$. L'abbé Châtel (1795-1857) fonde

46. Voir Pierre-Samuel Dupont de Nemours, Philosophie de l'univers, Paris, Impr. de Du Pont, 1793.

47. Philibert Audebrand, Michel Chevalier, Paris, 1861, p. 4, cité par Walter Benjamin, Paris, capitale du XIXe, Le Livre des passages, traduction française, Paris, Le Cerf, 1993, p. 608.

48. Martinès de Pasqually (?-1774) fonde l'ordre des chevaliers maçons élus coëns de l'univers, de forme maçonnique à vocation théurgique. Louis Claude de Saint-Martin (1743-1803) fut l'un de ses élèves. Jean-Baptiste Willermoz crée une maçonnerie spécifiquement chrétienne, héritière de la doctrine coën (régime écossais rectifié). Sur ce point, voir l'article de Christine Bergé.

49. Christine Bergé, "Saint Martin", dans Dictionnaire du monde religieux dans la France contemporaine, Les marges du christianisme, sectes, dissidences, ésotérisme, sous la direction de Jean-Pierre Chantin, Paris, Beauchesne, 2001, p. 216.

50. Alexandre-André Jacob (pseud. Alexandre Erdan), La France mistique [sic], Paris, Couton Pineau, 1855 , p. 55.

51. Louis Alphonse Cahagnet, Les Arcanes de la vie future dévoilés, Paris, Vigot, 1847. Le titre rappelle celui de l'ouvrage de Swedenborg: Les Arcanes célestes dont la traduction systématique des œuvres de Swedenborg par Le boys de Guay commence en 1847 mais le texte latin était fort connu. 
après sa rupture avec Rome en 1830 une Église catholique française, «la petite Église». Abel (ou Jean Simon) Ganneau (vers 1805-1851) organise une religion évadienne dont il se déclare en 1838 être le Mapah (MamanPapa) et se lie d'amitié avec Alphonse Louis Constant mieux connu sous son pseudonyme d'Eliphas Lévi-Zahed (1810-1875) ${ }^{52}$. L'émergence de ces nouvelles croyances pénètre, traverse et brise en bien des points la vieille croyance chrétienne. Elles proposent de nouvelles manières de penser l'univers et les relations entre microcosme et macrocosme. Charles Fourier est ainsi l'un des premiers à inventer une cosmogonie où les âmes se réincarnent ici et là dans l'espace des astres et nombre de ses contemporains, dont Boucher de Perthes et Camille Flammarion, croient à la pluralité des mondes habités.

Ces nouvelles formes de spiritualité inventent des relations avec un type de surnaturel non chrétien (ainsi celui de la croyance en la réincarnation ou en la vie sur d'autres planètes) que des visionnaires en état somnambulique confortent volontiers. C'est pourquoi l'Église catholique s'en inquiète. Si elle n'exclut nullement de son dogme ni même de ses marges l'intervention d'entités spirituelles, elle n'en demeure pas moins soit très prudente face à ces phénomènes, soit très hostile ${ }^{53}$. Dès la première décennie du XIX ${ }^{e}$ siècle, de nombreux écrits d'ecclésiastiques dénoncent dans ces pratiques la présence du diable ${ }^{54}$; n'oublions pas que la France redevient terre de mission au début de la Restauration et que les menaces proférées en chaire par les orateurs missionnaires sont vraiment infernales...

Si le somnambulisme magnétique dérange l'ordre catholique du premier retour à la monarchie (d'autant plus, répétons-le, que ces visionnaires sont toujours des femmes), il ne plaît pas non plus au nouveau pouvoir libéral mis en place par la Révolution de 1830, mais pour d'autres raisons. On sait que la monarchie doit, dès novembre 1831, faire face à la révolte des Canuts, puis à nouveau aux insurrections ouvrières de Lyon suivies de celles de Paris en avril 1834 , toutes réprimées de manière sanglante. On sait aussi que ces années 1830 sont irriguées par les projets des réformateurs sociaux, ceux de Charles Fourier et d'Henri de Saint-Simon en particulier ${ }^{55}$. Or si le magnétisme avait repris de la vigueur avec la première Restauration et le retour des émigrés qui l'avaient beaucoup pratiqué en exil, il a pris, sous la monarchie orléaniste, une ampleur populaire.

Cette pratique magnétique se mêle en effet bien souvent à d'autres théories médicales dissidentes, telle l'homéopathie ou la médecine de Raspail ${ }^{56}$,

52. Ce n'est que dans les années 1850 que ce dernier prend le pseudonyme d'Eliphas Lévi-Zahed (1810-1875) et professe un occultisme prétendant aux sciences positives.

53. Voir les travaux de David Armando, en particulier Il magnetismo animale tra scienza, politica e religione. Nuove fonti e ipotesi di ricerca, dans Laboratorio dell'ISFPF, II, 2005, 2, p. 10-30.

54. En particulier ceux de l'Abbé Wurtz

55. Voir Michèle Riot-Sarcey, Le Réel de l'utopie, essai sur le politique au XIXe siècle, Paris, Albin Michel, 1998.

56. Voir Dr Charpignon, Coup d'cil appréciateur sur certaines doctrines médicales, systèmes classiques, homéopathie, magnétisme etc.. Paris, Germer Baillière, 1849. 
souvent couplées à des méthodes d'éducation telles celle de Jacotot ${ }^{57}$ ou de Pestallozi ${ }^{58}$ en Suisse. Dans les critiques académiques adressées au magnétisme pendant ces années, ce n'est ainsi plus l'anti-aristocratisme qui s'exprime mais (non sans cynisme) le souci des pauvres filles du peuple qui deviendraient, sous la plume des adversaires du somnambulisme, des cobayes d'expérience qu'on pique et qu'on brûle pour démontrer leur anesthésie, «les unes prises dans un hôpital, les autres dans un modeste atelier d'ouvrières " ${ }^{59}$. Or bien loin de cette présentation, les adeptes du magnétisme défendent au contraire l'idée non seulement de l'appropriation du savoir de guérison (en particulier par les mères), - le magnétisme devenant une «médecine populaire, rivale de celle des écoles " ${ }^{60}$ - mais d'un développement d'une sociabilité nouvelle créée par cette «sympathie» magnétique entre les êtres. De petites sociétés se constituent en effet bien souvent autour d'un magnétiseur et de sa somnambule qui élaborent de nouvelles relations sociales, s'inspirant parfois de Fourier ou de Swedenborg ${ }^{61}$ et de bien d'autres encore.

En 1839, paraît en France un ouvrage qui se donne comme écrit par un ecclésiastique italien, le Père Scobardi, traduit par un médecin français ${ }^{62}$. L'ecclésiastique paraît y dénoncer violemment les méfaits du magnétisme dont les pratiques ébranleraient la société et mettraient en question "L'ORDRE social tout entier» ${ }^{63}$. Il rapporte ainsi que Mgr l'évêque de Moulins s'est élevé en chaire "contre ces ténébreuses inventions, ces mystérieuses découvertes des prétendus savants modernes, adeptes de matérialisme et corrupteurs de la morale [...] " ${ }^{64}$ soulignant particulièrement la lubricité des regards... Il exhorte également M. de La Marne, l'un des rédacteurs de L'Éclair, journal religieux fort à la mode, à faire de son mieux pour amener le gouvernement français à lui prêter son appui dans son combat. Ce journaliste avait en effet publié en 1828 une Étude raisonnée du magnétisme et Preuves de l'intervention des puissances infernales dans les phénomènes du somnambulisme magnétique ${ }^{65}$. Pour finir, Scobardi met en garde contre le matérialisme et «la vaine science des hommes», et ajoute : "Ce qui prouve clairement qu'il suffit de pendre les imprimeurs, les philosophes, les phrénologistes et les magnétiseurs pour

57. Voir Jacques Rancière, «Savoirs hérétiques et émancipation du pauvre» dans Les sauvages dans la cité. Auto-émancipation du peuple et instruction des prolétaires au XIXee siècle, Paris, Champ Vallon, 1985, p. 34-53.

58. Dont Allan Kardec, alias Hyppolite Denizard Rivail, le fondateur de la religion spirite est un des élèves et admirateur ensuite en tant qu'instituteur lui même.

59. Claude Burdin et Frédéric Dubois (d’Amiens) Histoire académique du magnétisme animal, Paris, Baillière, 1841, p. 94. Le livre de 651 pages est une somme contre le magnétisme.

60. Dr Charpignon Coup d'eil..., ouv. cité, p. 37.

61. Voir Nicole Edelman, Voyantes..., ouv. cité, p. 66-70.

62. Rapport confidentiel sur le magnétisme animal et sur la conduite récente de l'Académie royale de médecine, adressé à la congrégation de l'Index et traduit de l'italien du R. P. Scobardi par Ch. B***. D.-M.-P (docteur en médecine de Paris), Paris, Dentu, Germer Baillière, 1839.

63. Idem. p. 77. Les majuscules sont dans le texte.

64. Rapport confidentiel sur le magnétisme animal..., ouv. cité, p. 77. Les italiques sont dans le texte.

65. Étude raisonnée du magnétisme et Preuves de l'intervention des puissances infernales dans les phénomènes du somnambulisme magnétique, in $8^{\circ}, 32$ p., Paris, 1828. 
changer la terre en véritable paradis. " ${ }^{66}$ Cette dernière phrase, par son excès même, dévoile la supercherie : ce livre est en réalité un pamphlet écrit par un médecin favorable au magnétisme et le ridicule caricatural des critiques a pour but de disqualifier les anti-magnétiseurs. Ces propos nous dévoilent cependant l'existence de craintes certainement réelles d'une société bien pensante.

À toutes ces raisons de condamner le magnétisme animal comme dangereux socialement et idéologiquement, il faut ajouter enfin qu'il pose de réels problèmes à la science médicale, comme on le lira dans plusieurs articles de ce numéro. L'état somnambulique fait en effet surgir ce que nous nommerions un savoir psychique de l'inconscient, savoir qui n'est ni pensé ni même pensable à la fin du XVIIIe siècle, pas plus que dans les premières décennies du XIX ${ }^{e}$ siècle; le seul inconscient concevable étant alors ce que nous appelons l'inconscient cérébral. Au-delà des raisons politiques et sociales propres à la France, là réside sans doute la raison principale de cette mise à l'écart précautionneuse du magnétisme animal par la science médicale académique partout en Europe sans pour autant qu'il y soit condamné comme en France.

Partout en Europe et en Amérique du Nord, les travaux et recherches sur le magnétisme se poursuivent cependant ouvertement ou en catimini: tâtonnements des thérapeutiques par les médecins des eaux thermales qui y pratiquent massivement le magnétisme, expériences de mise en état de somnambulisme réalisées par des médecins non spécifiquement magnétiseurs ou encore, en manière de jeux, par des internes dans des hôpitaux sur des malades (surtout des femmes) ${ }^{67}$. Tout cela conduit à l'observation attentive des effets anesthésiques du somnambulisme et à la nouvelle dénomination de cet état par James Braid sous le terme d' hypnose en $1843^{68}$. Le magnétisme animal ouvre ainsi partout, et pour des décennies, de nouvelles questions auxquelles des réponses finissent par être données avec la naissance de la psychologie comme discipline, puis de la psychanalyse.

Nicole Edelman est maître de conférences (HDR) en histoire contemporaine à l'Université Paris Ouest-Nanterre

66. Idem, p. 144.

67. En particulier ceux qui traitent les hystériques : voir Nicole Edelman, Les métamorphoses de l'hystérique. Du début du XIXè siècle à la Grande guerre, Paris, La Découverte, 2003.

68. James Braid, Neurypnology or the Rational of Nervous Sleep, London, J. Churchill, 1843. 